\title{
Nein zu unbefristeter Steuerung, aktuelle Regelung ist akzeptabel
}

\author{
Jürg Schlup \\ Dr. med., Präsident der FMH
}

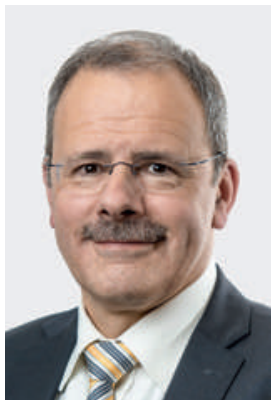

Es ist unbestritten, dass die Kostenentwicklung im Gesundheitswesen eine zentrale Herausforderung der Zukunft darstellt. Der Anteil der über 65-Jährigen und ganz besonders die Gruppe der über 80-Jährigen werden in den kommenden Jahren deutlich wachsen. Gleichzeitig nehmen die medizinischen Möglichkeiten zu und die Ansprüche an die Gesundheitsversorgung steigen. Die Erhöhung der Nachfrage nach Gesundheitsleistungen ist also gewiss.

Statt innovative Konzepte zur bestmöglichen Bewältigung dieser Entwicklung zu erarbeiten, versucht die Politik nun bereits seit 2002 der steigenden Nachfrage durch Beschränkungen ambulanter Arztpraxen Herr zu werden - ein von vornherein irrationaler Ansatz: praxisambulant tätige Ärzte verursachen $14 \%$ der Gesundheitskosten, behandeln aber den grössten Teil der Erkrankungen. Zudem verlief die Kostenzunahme im praxisambulanten Sektor immer proportional zu der Kostenentwicklung des gesamten Gesundheitswesens und zeigte keinen spezifischen Handlungsbedarf.

So verwundert nicht, dass die erhoffte kostendämmende Wirkung der bisherigen Zulassungssteuerung auch nach dreizehn Jahren unbelegt bleibt. Erreicht wurde lediglich eine Verschiebung der Inanspruchnahme von Leistungen und der damit verbundenen Kosten in den spitalambulanten Sektor, wie dessen deutliche Ausdehnung seit 2002 zeigt.

Eine Begrenzung des Angebots bei steigernder Nachfrage ist irrational und verlagert die Kosten - statt sie zu reduzieren.

Trotz fehlender Kostenwirkung plant der Bundesrat nun die dauerhafte Verankerung der Zulassungssteuerung des ambulanten Sektors im KVG Art. 39 und 40: Die Kantone sollen im Fall einer - durch sie selbst zu definierenden - «Überversorgung» sowohl die Neuzulassungen ambulant tätiger Ärzte als auch deren Arbeitspensen begrenzen dürfen. Die bereits bestehenden zahlreichen Rollen- und Interessenkonflikte der Kantone als Gesetzgeber, Kontrollbehörde, Ausbildner, Spitaleigentümer, Spitalplaner, Spitalbauherr, Spitalbetreiber, Spitalteilfinanzierer, Tarifgenehmigungsbehörde und Schlich-

tungsstelle für Tarifstreitigkeiten würden somit noch erweitert. Als Steuerungsbehörde für ambulant tätige Ärzte könnten die Kantone z.B. die Konkurrenz zu ihren Spitalambulanzen weitgehend begrenzen. In den Spitälern notwendige Änderungen - wie z.B. die Schaffung von mehr Teilzeitstellen - könnten sie schlicht ignorieren, da angestellten Ärzten zum Spitalarbeitsplatz - abgesehen vom Berufsausstieg - kaum eine Alternative bliebe. Die nicht zuletzt auch vom Bundesrat geteilte Forderung nach «zeitgemässen Versorgungsmodellen» würde damit konterkariert.

Die vorgesehene Zulassungssteuerung löst folglich keines der bestehenden Probleme, hemmt wichtige Ent-

Die vorgeschlagene Zulassungssteuerung löst keines der bestehenden Probleme und hemmt wichtige Entwicklungen.

wicklungen und schafft Unsicherheit bei der unternehmerischen Planung im praxisambulanten Sektor. Zudem steht im Februar 2017 mit der Umsetzung der Masseneinwanderungsinitiative die Kontingentierung von Arbeitskräften bevor, auch im Gesundheitswesen und für Ärzte. Es ist nicht nachvollziehbar, warum der Bundesrat per Juli 2016 noch eine Steuerung einführen will.

Gleichzeitig verzichtet der vorliegende Gesetzesentwurf auf das bewährte Qualitätskriterium der heutigen, seit 2013 geltenden Regelung: Zugelassen sind Ärztinnen und Ärzte, welche mindestens drei Jahre an einer schweizerisch anerkannten Weiterbildungsstätte gearbeitet haben. Die Weiterführung dieser Regelung wäre für die FMH akzeptabel. Für eine hohe Patientensicherheit ist entscheidend, dass Ärztinnen und Ärzte mit dem Schweizer Gesundheitswesen vertraut sind und eine qualitativ hochstehende Weiterbildung durchlaufen haben.

Die vorgeschlagene Änderung des KVG hingegen lehnt die FMH - der Zentralvorstand sowie die Delegiertenversammlung einstimmig - ab und behält sich die Referendumsmöglichkeit vor. Wenn wir unseren Versorgungsstandard halten möchten und dieser volkswirtschaftlich finanzierbar sein soll, benötigen wir einen Ausbau des ambulanten Bereichs und hochmotivierten ärztlichen Nachwuchs - hierfür gilt es Anreize statt Hürden zu schaffen. 